\title{
The Analysis of Application of Huger Marketing in China's Mobile Phone Industry
}

\section{Qinyuan Chen}

School of Management, Hunan University of Science and Technology, Xiangtan, China

Email address:

yuan7958@126.com

\section{To cite this article:}

Qinyuan Chen. The Analysis of Application of Huger Marketing in China's Mobile Phone Industry. Science Innovation. Vol. 4, No. 2, 2016, pp. 117-121. doi: 10.11648/j.si.20160402.27

Received: February 29, 2016; Accepted: March 23, 2016; Published: May 11, 2016

\begin{abstract}
Facing the increasingly fierce mobile phone market competition, all businesses are looking for the breach of the competitive advantage to attract consumers by taking a new way of marketing.In order to get more profits and higher market share, businesses turn their attention to "hunger marketing", especially in the mobile phone business. The phenomenon of Chinese mobile phone companies use hunger marketing is becoming more common,and there are many companies success. In this paper, we use instance analysis, supplemented by literature analysis method. By analyzing the Chinese mobile phone companies to use the example of hunger marketing, summarize the effect of hunger marketing brings to the mobile phone companies and the conditions for adopting the hunger marketing success. Then Suggestions are put forward to the mobile phone enterprises for practical application of "hunger marketing".
\end{abstract}

Keywords: Utility Theory, Hunger Marketing, Consumer Psychology

\section{饥饿营销在中国手机企业的应用分析}

\section{陈沁园}

管理学院, 湖南科技大学, 湖南湘潭, 中国

\section{邮箱}

yuan7958@126.com

\begin{abstract}
摘要：面对手机市场日益激烈的竞争，各商家纷纷寻找竞争优势的突破口，通过采取新的营销方式来吸引消费者，为 了获得更多的利润和更高市场占有率，商家们开始将目光投向了 “饥饿营销” 这种营销方式，在手机企业尤为明显。 中国手机企业运用饥饿营销的现象越来越普遍, 并且也有很多企业取得了成功。本文主要运用实例分析法, 辅以文献 分析法。通过分析中国手机企业运用饥饿营销的例子，总结饥饿营销给手机企业带来的影响及成功运用饥饿营销的条 件。进而对手机企业“饥饿营销” 的实际运用提出建议。
\end{abstract}

关键词: 效用理论, 饥饿营销, 消费者心理 


\section{1. 引言}

\section{1. 研究背景}

随着科技的进步, 经济的发展, 我们身边的大多数 人都换上了智能手机。几年前, 大家使用的手机以诺基 亚, 三星等国际品牌为主。但现在可以看到大家使用的 智能手机品牌众多, 其中不乏小米、华为这样的中国国 产手机品牌。中国手机制造业已经有近20多年的历史, 众多企业逐渐进入手机行业, 虽然国际企业实力最为突 出, 但中国手机企业也在迎头赶上。今天, 手机种类更 加多样化与个性化, 它不仅仅是通话机器, 它更代表一 种服务, 一个产业链。随着中国手机企业总数和手机普 及率的增长, 移动电话用户增长变缓, 企业的竞争空前 激烈。在这种情况下，手机企业纷纷使用营销手段来提 高竞争力, 在这之中, 小米手机依靠 “饥饿营销” 获得 了空前成功。

\section{2. 研究目的和现实意义}

饥饿营销作为时下较为 “时髦” 的营销手段, 许多 手机品牌通过运用它获得了突破性的发展。如何正确得 运用它来让企业发展的更好是一个极具现实性与时代感 的话题。本文通过对中国手机企业具体应用 “饥饿营销” 分析, 进而分析饥饿营销对手机企业的影响以及饥饿营 销的应用条件。在此基础上提出饥饿营销在中国市场的 应用建议, 把部分手机企业的成功经验提炼出来, 供其 他手机企业参考。

\section{3. 国际研究现状}

在国际上，20世纪已经有学者对 “饥饿营销” 的消费 心理进行研究。Fromkin (1973) 认为, 当消费者产生 “希 望与众不同的” 需求时, 为了追求这种与众不同的感觉而 去努力获得这种稀缺产品。Brehm Sharon（1996）提出了 心理抵抗理论, 该理论提出消费者为了抵抗某种产品的稀 缺所带来的现有购买自由的限制与丧失, 会对该产品产生 较之前更为强烈的拥有欲望和冲动。

V Shankar和S Balasubramanian (2009) 在《Mobile marking:a synthesis and prognosis》中通过对国际手 机市场深入的研究, 详细的描述了手机营销活动的策划和 实施方法, 并从营销组合的角度, 全面概述了企业应该制 定的一些营销活动。

21世纪起，中国国产手机开始起步。刘金峰和文亚 青（2011）发表了《论 “饥饿营销” 策略的负面影响和 实施条件》一文。文章从饥饿营销实施的条件和带来的 影响两方面做了阐述。邓璐楠（2012）发表《社会化媒 体发展背景下的小米手机营销策略研究》一文。文章研 究了在社会化媒体发展的背景下小米手机的营销策略。 总结了小米手机营销的经验, 为其他企业开展社会营销 提供有力参考。

\section{2. 饥饿营销理论综述}

\section{1. 饥饿营销的概念}

饥饿营销是指商品提供者有意调低产量, 以期达到 调控供求关系、制造供不应求 “假象”、维持商品较高 售价和利润率的营销策略。在西方经济学中存在 “效用 理论” ，是指消费者从对商品和服务的消费中所获得的 满足感, 它属于一个心理概念, 具有主观性, 在市场营 销中是被称为 “饥饿营销”。饥饿营销常应用于商品或 服务的商业推广中。

现实生活中常有一些这样的现象, 买房连夜排队等候 抢房、买车也要先交订金排队等候、网络上各种 “限量版 秒杀” 活动更是层出不穷。这不禁让人疑问, 为何在物质 条件越来越丰富的今天, 买东西反而这么难呢? 事实上, 这只是一个商业手段, 企业故意刺激消费者的购买欲望, 同时却不及时满足, 使消费者产生更强的购买欲望, 造成 供不应求的市场现象。

\section{2. 饥饿营销策略的特征}

\subsection{1. 较强的宣传力度}

在产品推入市场前, 一般会先运用网络、电视、杂志 等媒体对产品进行大力宣传, 制造出可以激发顾客购买欲 的 “消费点” ，却在产品具体信息上保持一定神秘性。从 而勾起消费者的好奇心，使消费者产生一种迫切想要得到 该产品的心理, 为新产品上市做好准备。

\subsection{2. 合适的时机}

企业的营销策略一方面受市场环境的影响，另一方面 受竞争对手营销策略变化的影响。企业一般会根据市场的 波动和竞争对手的行销策略来选取合适的时机, 制定营销 策略。

\subsection{3. 推广有针对性}

如果想依靠 “饥饿营销” 策略获取成功, 通常要根据 产品的特色、渠道等各方面因素, 针对产品的受众来进行 特定的推广。

\subsection{4. 人为造成供不应求的现象}

销售商和厂商利用营销手段激发消费者的兴趣和购 买欲望后, 人为减少产品的供应量。使顾客在进行购买时, 出现因购买者 “过量” 而造成的供不应求的状态。而消费 者为了获取该产品, 不得不提前预定购买。

\section{3. 饥饿营销在国际上手机企业的典型应用}

说到饥饿营销在手机行业的应用, 大家最先想到的就 是“iPhone”。从2004年至iPhone面世前的两年多时间里, 它的全部细节完全保密。这极大地引起了媒体及消费者对 它的渴望, 外界对其外观和名字乃至销售模式都有着各种 各样版本的猜想。从而, 关于iPhone的讨论不绝于耳。 
图表显示, iPhone在2007年推出之后, 销量仅为 136 万台, 2009年, 新款手机推出之后, 销量达到2073万台。 万台, 到了2008年, iPhone销量已经翻了十倍, 达到了 1163

表1 iNotes4You网站公布iPhone 2007-2015年的销售数据。

\begin{tabular}{llllllllll}
\hline 年份 & 2007 & 2008 & 2009 & 2010 & 2011 & 2012 & 2013 & 2014 & 2015 \\
\hline 销售量 (万台) & 1.36 & 11.63 & 20.73 & 39.99 & 70.29 & 125.05 & 150.26 & 169.22 & 231.22 \\
同比增长率 (\%) & & 755.15 & 78.25 & 92.91 & 75.77 & 77.91 & 20.16 & 12.62 & 36.64 \\
\hline
\end{tabular}

当然, 苹果这样的宣传策略也是基于其强大的品牌号 召力和过硬的产品实力之上的。事实证明, 苹果的饥饿营 销取得了很大的成功, 从iPhone问世到今天, iPhone热从 未退烧。不分国界,一有新的产品上市, 参与抢购的果粉 们比比皆是。

\section{3. 饥饿营销在中国手机企业的典型应用分析}

随着手机企业的增加, 用户增幅减缓, 手机企业竞争 的愈发激烈, 有效的营销手段成为手机产品增强竞争力的 重要方式。手机企业如何突围? 中国不少手机企业都采取 了饥饿营销这一手段, 并取得了良好的成效。以下选取三 个中国手机生产公司运用饥饿营销的例子进行分析。

\section{1. 北京小米科技有限责任公司}

小米公司可以称得上是中国第一家运用饥饿营销的 手机企业。像iPhone一样, 它也遵循了 “发布会一上市日 期公布一等待一上市新闻报道一用户排队抢购一全线缺 货” 的销售路径。

在2011年8月以前, 中国还没有通过官方网站直接销 售手机的案例, 充其量是通过淘宝、京东第三方网站来销 售手机。现实中最普遍的销售方式还是通过店铺商场销售。 此时, 小米公司大胆得采用了官网直销的模式。从2011 年的 30 万台到 2015 年的 6000 万台, 小米手机的关注度与销 量都不可同日而语。但 “不抢购, 无小米” 的销售方式在 业界一直存在较大争议。

\section{2. 华为技术有限公司}

当小米手机运用饥饿营销取得巨大成功之时, 华为手 机亦不堪落后。2010年, 华为首次入围美国《财富》杂志 世界500强。华为在2013年12月16日召开发布会, 发布了 荣耀系列, 荣耀品牌的运营是独立于华为其他系列手机, 销售渠道的拓展主要依托于电商, 高性价比和华为品牌的 拉力使得荣耀品牌刚推出就收获良好的市场反馈。

但从华为荣耀的火爆不难看出华为的现货产量跟不 上市场需求。华为手机发货严重延迟的问题、供货不足等 问题遭到了广大消费者的吐槽。这些问题至今仍未解决。 华为目前出现市场热情已经被唤起, 却无货可供的局面。

\section{3. 中兴移动通讯有限公司}

中兴旗下多款手机采用了预约抢购的营销手段, 效果 都十分明显。从艾瑞咨询的品牌关注度调查显示, 中兴品 牌位居第 15 位, 仅为 $6.3 \%$ 。手机的品牌形象始终是中低端 手机的代表。2011年, 中兴不断在智能机市场上发力, 以 多款明星机型实现了智能终端销售的快速增长, 成为苹果
之后增速最快的智能手机厂商按出货量计第四季度上升 成为全球第四大手机厂商。

这几年来, 中兴出货量大幅增加, 这离不开饥饿营销 的运用。不过中兴做出承诺, 这些预约抢购机型在发售起 一个月内都将提供现货，不再需要抢购。目前中兴新出的 机型都已经做到这一点。中兴这一做法得到了消费者的称 赞。

\section{4. 中国手机企业应用饥饿营销后带来的影响}

饥饿营销是一把双刃剑。就短期来看, 饥饿营销能够 增加产品的销量, 但是长期而言, 饥饿营销会让消费者产 生质疑, 甚至消耗掉消费者的耐心。最终有可能将顾客送 给对手。从现实来看, 饥饿营销有弊有利, 应该辩证看待。

\section{1. 实施饥饿营销给手机企业带来的积极影响}

\subsection{1. 提高产品销量, 增加企业收入}

由小米提供的官方数据看, 小米手机自 2011 年出世以 来, 正以惊人的速度发展着。

表2 小米手机2011-2014的总体销售数据。

\begin{tabular}{|c|c|c|c|}
\hline 年份 出货量 (万台) & 出货量增长率 & 销售额（亿元） & 销售额增长率 \\
\hline 201130 & & 5.5 & \\
\hline 2012719 & $2400 \%$ & 126. 5 & $2300 \%$ \\
\hline 20131870 & $260 \%$ & 316 & $250 \%$ \\
\hline 20146112 & $327 \%$ & 743 & $235 \%$ \\
\hline
\end{tabular}

\section{1.2. 放大产品及品牌的号召力, 提高顾客忠诚度}

当消费者被产品的外形、性能吸引后, 他们会关注和 宣传产品讯息。他们非这种产品不买, 只认这个品牌, 忠 诚度提高。当消费者身边的人天天都在排队抢购、讨论某 个产品, 乃至成团地与对手的粉丝争论时, 用户的这种自 觉宣扬产品的营销力是不容忽视的。消费者就容易受身边 的人影响, 从而采取和他们相同的行为一一也关心这个产 品或品牌, 对品牌的信赖度和忠诚度因此获得进一步提升。

\section{1. 3. 有利于塑造良好的品牌形象, 提高品牌关注度}

产品的热销会给消费者传递这样一种讯息: 这种产品 很好, 否则不会缺货, 这种产品值得购买, 物有所值。再 加上良好的口碑。打造出了一个值得信任的品牌形象。

\section{2. 饥饿营销给手机企业带来的负面影响}

\subsection{1. 消耗顾客耐心, 将客户送给竞争对手}

实施 “饥饿营销” 的本质是让消费者在消费过程中得 到的满足感。但是, 效用具有主观性, 不同于物质的使用 
价值。因此, 如果企业在实施饥饿营销过程中没有把握好 度, 很容易造成 “物极必反” 的效果。如: 消费因受低价 吸引产生了强烈的欲望, 但供应量太少, 消费者在期望的 时间里难以获得该产品, 或者加价过多超出消费者的承受 范围, 此时消费者会认为这款产品可望不可求。从而选择 其他同类产品来替代。

\subsection{2. 低企业信用形象}

由 “饥饿营销” 的实施过程可以看出, 企业实施饥饿 营销启示是利用买卖双方信息不对称这一条件, 实现对市 场供求状况的控制。本质上是一种企业对市场的供给和需 求的有意识的控制, 售前, 销售控制活动。这与诚信营销 是相悖的, 如果企业长期使用饥饿营销, 当消费者长期得 不到满足时, 他们就会冷静下来, 理智地思考。他们会主动 搜集信息, 根据信息来分析饥饿营销的实质, 一旦觉察产 品的供不应求是商家人为制造的, 就会认为自己受到欺骗, 从而不再信任企业。

\subsection{3. 拉长了产品的销售周期}

不难看出, 饥饿营销通过拆分分批次销售产品, 拉长 了产品的销售周期。这种做法机具风险性, 一方面, 它让 企业收回成本的周期变长; 另一方面, 它给了竞争对手模 仿的时间, 会缩短自己产品保持优势的时间, 容易给竞争 对手可趁之机, 继而丧失主动权。

\section{5. 实施饥饿营销的应用条件分析}

\section{1. 具备一定的品牌号召力}

品牌是实施 “饥饿营销” 的产品基础, 品牌能够得到 用户的肯定是饥饿营销实施的重要条件。一般而言, 具有 广泛的知名度, 良好的口碑以及成熟度高的品牌更容易得 到消费者的认可。譬如华为、中兴等品牌都是在中国具有 较高知名度与认可度的品牌, 深得消费者的信赖。

\section{2. 产品质量优异, 综合竞争能力强}

设计和产品质量是决定市场竞争力的关键因素, 也是 产品获得不可替代优势的重要方式, 是企业成功实施 “饥 饿营销” 的 “资本”。设计独特、质量好的产品, 不但可 以激发市场的潜在需求, 将现实需求放大, 甚至会使消费 者产生 “非它不买” 的想法。

\section{3. 消费者消费心理不成熟}

饥饿营销能否良好的开展受消费者消费心理的影响。 当前中国市场中, 基本不存在彻底理性的消费者, 消费者 常常受各种心理因素的影响。比如求同、求新、攀比、好 奇等。

求同即常说的从众心理, 当大家都在消费一种产品时, 消费者会在周围人消费行为的带动下去购买。

求新动机追求产品的创新性和时尚性。现在年轻人大 多追逐新鲜与时尚。对于手机, 很多人都在不断追求时下
最新潮的产品, 十分关注新产品, 为此不惜耗费大量的物 力、财力。企业常常利用这种心理来开展饥饿营销。

攀比心理, 对于时下流行的产品, 人与人之间可能会 存在 “你有, 我也一定要有” 的这种心理, 不甘心落于人 后, 哪怕预定或排队等候也心甘情愿。

消费者的购买欲会因为好奇心理而更加强烈。所谓, 得不到的才是最好的。企业运用饥饿营销的第一步就是勾 起消费者的兴趣, 但又保持产品的神秘感, 这就是利用了 消费者的好奇心理。

这些消费心理普遍存在于消费者中, 这是正常的。但 如果这些心理被营销者利用, 消费者受营销策略影响明显。 则反映当前消费者心理状态并不够成熟, 消费行为并不完 全理智。正是这些为企业实施 “饥饿营销” 策略提供了 条件。

\section{4. 市场竞争不充分}

当中国手机企业竞争充分时, 行业内会存在许多替代 品。当其中一种价格上升或者某种商品难以获得时, 消费 者仍然拥有主动权, 完全可以选择其他产品。目前, 手机 市场竞争并不充分。为什么小米手机那么难抢, 大家仍趋 之若鷔呢, 因为市场中和小米手机同价位的手机在性能上 都不如它, 小米手机的替代品较少。当市场中充满着像小 米一样物美价廉的手机时, 小米的饥饿营销将不再具有效 果。所以, 产品能够成功运用饥饿营销的重要前提是市场 竞争不充分。

\section{6. “饥饿营销” 在中国手机企业运用的经验借鉴}

\section{1. 手机研发采用用户参与模式}

小米手机广泛采纳用户的意见，让用户参与到小米手 机系统的研发中。相比其他的Android操作系统, 小米手 机界面、图标等别具一格, 使小米手机快速获得了手机发 烧友的认可。魅族手机自主研发的操作系统也采用许多消 费者的建议, 得到大众认可。其他手机企业在研发时也可 以学习这种良好的的方式, 进一步的发展自主创新, 开发 出更适应中国手机用户的硬件配置和功能。

\section{2. 宣传造势是保障}

饥饿营销成功的重要前提是良好的宣传造势。在产品 上市之前可以各种平台来开展宣传, 勾起消费者的好奇心, 俗称 “吊胃口”。饥饿营销成效的好坏, 与宣传的平台、 时间、手段密不可分。制造氛围也十分重要, 当产品出现 排队抢购、缺货、销售一空等现象时, 可以通过媒体进行 实时报道, 以渲染火爆、供不应求的氛围。在宣传过程中 需要格外留意的是信息传播的度。不能太少, 否则难以勾 起消费者和媒体的兴趣; 也不能太多, 否则会丧失产品的 神秘感。”吊胃口” 的度更需要小心把握, 太小则得不到 预期的效果，太大则易产生发效果，吓走消费者。 


\section{3. 把握合适的周期与准确的时机}

信息不对称、产品具有独特性是 “饥饿营销” 实施的 基础。在一种产品进入市场的初期, 企业缺少完全公开产 品信息的压力。因为在这一阶段, 市场中同类竞争产品较 少, 消费者缺乏了解产品信息的其他方式, 对产品的了解 十分有限。但在产品进入市场一段时间后, 竞争者会逐步 出现, 他们也会广泛宣传, 利用产品优势来吸引消费者。 消费者此时能够有多种渠道来了解多个产品的信息。此时, 企业必须公开更多的产品信息, 充分显示产品的优势。由 此可以看出信息不对称是暂时的, 其实产品的独特性也是 暂时的。这是因为, 当企业产品的独特性为公众所知时, 竞争对手会尽其所能地模仿。而随着模仿者的增加, 产品 将不具独特性。所以, 企业需要把握最佳时机在合理的周 期内实施饥饿营销。

\section{4. 准确预测市场对产品的容量}

企业实施 “饥饿营销” 的根本原因是增加收益。而企 业的收益是由价格和销售量共同决定的，当价格一定时， 销售量决定利润。商品的供求规律表明价格与需求量成反 比。要想实现利润目标, 在价格上必须严格控制, 因为消 费者对商品的价格会有一个容纳量。价格上涨后, 如果消 费者对这个价格的接受量的降幅多余价格的涨幅, 企业就 无法实现利润目标。所以, 在实施饥饿营销之前应进行广 泛的市场调研。科学推算、准确预测市场对产品数量和价 格的容纳量, 采用最合理定价及出货量。

\section{5. 完善售后服务与客户体验}

饥饿营销的销售渠道大多是采用商对客模式, 在官网 和第三方网站进行直销。毫无疑问, 这大大减少了企业的 运营成本。但从顾客反应可以看出, 售后服务是小米手机 等的软肋, 它多次遭到消费者的抱怨。消费者越来越发注 售后服务, 尤其是手机等非快销品。因此, 就长期而言, 企业必须完善售后服务体系建设, 让消费者放心消费。并 且, 实行饥饿营销的手机品牌大多缺少良好的体验渠道, 这导致顾客难以拥有全面的商品体验。建立直营店, 不失 为解决以上问题的一个办法。

\section{7. 结论}

本文对中国国产手机企业采用饥饿营销获得成功的 事例进行了描述说明。根据市场的反应, 分析得出饥饿营 销有利有弊, 适当的运用能够帮助企业发展, 但长期使用 会流失顾客, 损坏品牌。接着, 根据以上事例从产品本身、 消费者心理、市场环境等方面对饥饿营销成功应用的条件 进行了分析, 是有参考价值的。最后, 根据这些企业成功 运用饥饿营销的经验, 文章认为手机企业应注意以下几点: 手机研发采用用户参与模式、加强品牌创新、有效的宣传
造势、把握合适的周期与准确的时机准确预测市场对产品 的容量、完善售后服务与客户体验。

\section{参考文献}

[1] 赵根良. 基于消费者心理的饥饿营销策略 [J]. 河北北方学 院学报:社会科学版, 2012, 28(4): 48-55。

[2] 刘金锋; 文亚青. 论 “饥饿营销” 策略的负面影响和实施条 件 [J].广东石油化工学院学报, 2011, 21 (5) :69-78。

[3] 吕晓焕; 黄贺飞. 浅谈B2C直销模式下的限量营销--以小米 手机为例 [J]. 现代商业, 2013, 13:94-95。

[4] 洪昕; 王玨; 林花. 我国智能手机营销策略分析 [J]. 企业经 济, 2012, 08:85-86。

[5] 祝源; 汪莉; 豆传秀. 浅析 iPhone的营销策略 [J]. 商业文化 (上半月) , 2012, 01 。

[6] 欧阳岗. 旅游企业网络营销策略探析一基于小米手机网络 营销的比较 [J]. 旅游纵览（行业版）, 2012, 04:75-76。

[7] 张娇娇. 苹果产品的营销得失 [J]. 商业现代 化, 2013, 02:75-76。

[8] 于斌. 饥饿的小米 [J]. 商界（评论）, 2012, 02:126-129。

[9] 一帆. 饥饿的 “米粉”-- “网络饥饿营销” 的经典案例 [J]. Shanghai economic, 2013, 21: 29-31.

[10] 刘清华. “饥饿营销” 的应用条件分析 [J]. 现代营销 (学苑 版）, 2011, 06:64-65。

[11] Carlo M. The decline of a fully matured economy [J]. The Economic History Review, New Series, 1952, 5(2): $178-184$ 。

[12] Philip Kotler, Kevin Lane Keller. Marketing Management [M]. 清华大学出版社, 2011:233-251。

[13] 任天飞. 市场营销学 M]. 长沙: 国防科技大学出版 社, 2004:136-167, 188。

[14] Jerome E. McCarthy, Basic Marketing [M]. 上海人民出 版社，2001：43-48。

[15] Don E. Schultz, Philip J. Kitchen. Communicating globally: An Integrated Marketing Approach [M]. 机 械工业出版社，2012：103-107。

[16] V Shankar; S Balasubramanian. Mobile marking:a synthesis and prognosis $[\mathrm{J}]$. Jounal of interactive marking. 2009, 23: 118-129. 